\title{
Autonomous Formation Selection For Ground Moving Multi-Robot Systems
}

\author{
Shuang $\mathrm{Yu}^{1}$ and Jan Carlo Barca ${ }^{2}$
}

\begin{abstract}
Multi-robot systems have many useful real world applications including disaster management, exploration and surveying. Formation control is critical in these contexts as the success of groups often depend on the ability to generate and maintain particular formation shapes. It is also important that a multi-robot system can evaluate and select appropriate alternative formations when an ideal formation cannot be upheld, particularly in dynamic real world scenarios. A distributed formation selection mechanism that addresses these issues by enabling groups of unmanned ground vehicles to autonomously select, scale and morph formation shapes when navigating through dynamic environments is presented in this paper. Experiments on non-holonomic ground moving robots demonstrate the suitability of the proposed technology.
\end{abstract}

\section{INTRODUCTION}

The research community has recently shown a growing interest in multi-robot systems (MRS) due to their highimpact application areas, including disaster management, environmental monitoring, exploration and surveying. The advancement of this technology has created a demand for distributed mechanisms that can monitor and regulate formation configurations, as the success of groups often depends on the ability to preserve appropriate geometric structures [1]. As a result, formation control mechanisms that enable MRS to generate and preserve formations have been widely studied [2], [3], [4], [5], [6]. However their ability to select, scale and morph formations autonomously in dynamic environments has received much less attention. It is therefore important to address this issue as most real world situations require continuous adaptation to unpredictable environments, and because it is desirable to minimize the load on human operators in MRS contexts [7].

Some efforts in addressing this problem have therefore been made. Relevant works include the: i) graph theory based techniques presented in [8], [9], which offer a theoretical framework for how formations can be scaled up and down, ii) method described in [10] which enable swarms of simulated robots to identify appropriate formation scales on the basis of the number of robots in the system, and iii) research presented in [11] that demonstrates how groups of nonholonomic ground moving robots can scale and morph formations autonomously. A drawback with the work presented in [8], [9], [10] is that only varying formation sizes are considered. This is not ideal as teams of robots are sometimes

\footnotetext{
${ }^{1}$ Shuang $\mathrm{Yu}$ is with Department of Electrical and Computer Systems Engineering, Monash University, Melbourne, Australia shuang . yu@monash. edu

${ }^{2}$ Jan Carlo Barca is with Clayton School of Information Technology, Monash University, Melbourne, Australia jan. barca@monash. edu
}

required to alter their formation shapes to accomplish a task (e.g. if the robots have formed a broad formation and must navigate through a narrow opening). The main drawback with the work presented in [11] is that the authors did not demonstrate that their robots can select alternative formation scales and shapes in a distributed manner.

Researchers have also sought inspiration from biological morphogenesis in their efforts to address the above problem. In [12] the authors present a morphogenesis inspired neighborhood adaption mechanism that enables groups of epuck robots to distribute across a pre-defined target pattern according to the number of robots in the system. However, they do not scale the actual pattern up or down when the number of robots do not fit the original pattern. This makes it difficult to maintain appropriate formations when the number of robots changes dramatically. The problem of selecting appropriate formation shapes is furthermore not addressed. In [13] a morphogenesis inspired adaptive pattern formation mechanism for e-pucks is presented. A drawback with this technique is that the generated formations are based purely on circular patterns. This is a problem as different tasks often require radically different target patterns. A more flexible technique that can respond effectively to a broad range of different scenarios is therefore required.

We address the above drawbacks by proposing a distributed technique that enable MRS to select, scale and morph formation shapes when navigating through dynamic environments. The proposed technology builds on the work presented in [11] and is validated on real non-holonomic ground moving robots.

The remaining parts of this paper are structured as follows; Section 2 offers an overview of the system design, along with detailed descriptions of the proposed formation control mechanisms. Section 3 describes results from experiments that have been designed to evaluate the proposed technology. Finally, conclusions and suggestions for future research are presented in Section 4.

\section{AUTONOMOUS FORMATION SELECTION}

In this research, we assume that the robots have been assigned from the outset an ideal formation for the task at hand and a series of alternative formations with various scales. Any two dimensional formation can be assigned to the robots as long as the robots are connected. We also assume that the robots are sometimes required to alter the ideal formation to successfully navigate through dynamic environments without experiencing collisions. Different formations have different characteristics that make them ideal for particular tasks, for 
example, a maximum coverage formation covers an area with the minimum number of robots, while a linear formation makes it possible to move through narrow openings. The ideal formation should therefore be selected according to the task and hand. Additionally, the MRS should always seek to preserve the ideal formation.

To achieve this aim, the proposed technique iterates through a four stage process that involves: 1) sensing and preprocessing, 2) local decision making, 3) distributed agreement and 4) navigation. In Stage 1, each robot collects information from its immediate surroundings by means of an on-board distance measurement sensor, and prepares this information for further processing. In Stage 2, the collected data is fed into a decision tree that enables each robot to identify a locally preferred formation. These locally preferred formations are then shared with neighbouring robots so that consensus can be reached on a group level in Stage 3. Each robot calculates its desired position in the new formation and navigates towards the target location in Stage 4 , if a formation change is required.

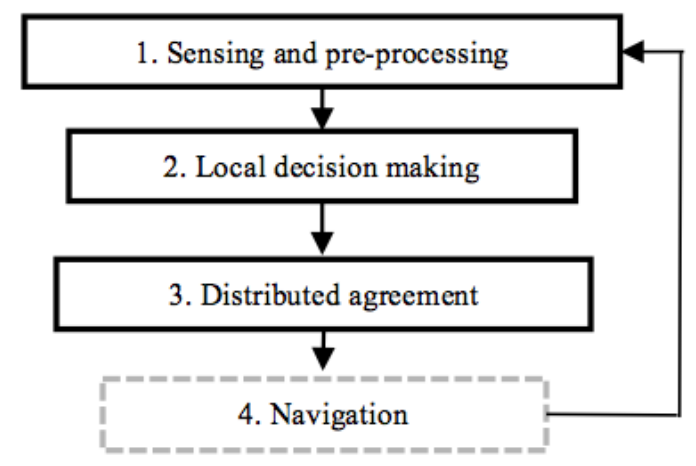

Fig. 1: System overview

An overview of the proposed technique is given in Fig. 1. Detailed descriptions of Stages 1 through 3 are provided in the following subsections. The reader is referred to [11] for a detailed description of Stage 4.

\section{A. Sensing and Pre-Processing}

In Stage 1 distances to obstacles that appear within sensing radius $r_{s}$ of robot $R_{0}$ are collected by means of an onboard distance measurement device with swipe angle $S_{\text {angle }}$. If an obstacle is detected within this region, it is regarded as blocking the path of $R_{0}$, and further information about the surrounding obstacles and robots is obtained.

To collect information about the surrounding obstacles and robots, we first define the forward direction for $R_{0}$ as the $\mathrm{y}$-axis of the robot's orientation, and the $\mathrm{x}$-axis as being perpendicular to the forward direction, as illustrated in Fig. 2. With $N$ obstacles and $M$ robots within the sensing region of $R_{0}$ we also define relative angles $\theta_{o b s, i}$ to the surrounding obstacles, and $\theta_{r, i}$ to the surrounding robots. The Euclidean distance $d_{o b s, i}$ to each obstacle and $d_{r, i}$ to each surrounding robot is then projected onto the $\mathrm{x}$-axis of $R_{0}$ according to

$$
d_{o b s, i}^{\prime}=d_{o b s, i} \sin \left(\theta_{o b s, i}\right)
$$

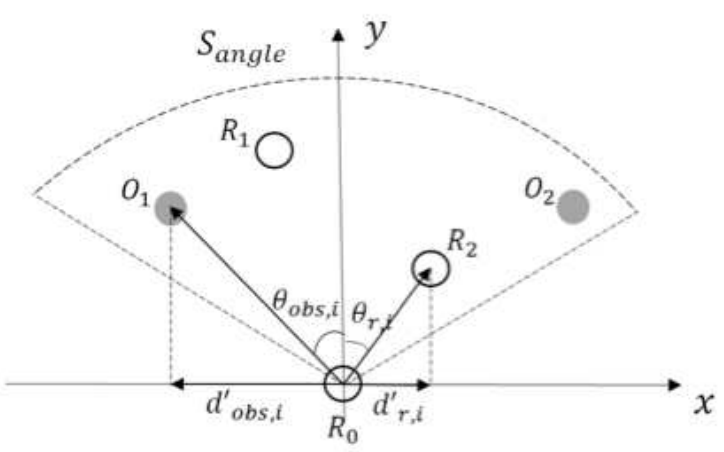

Fig. 2: Calculating the projected distance between the closest obstacle and the closest robot

$$
d_{r, i}^{\prime}=d_{r, i} \sin \left(\theta_{r, i}\right)
$$

to determine if there is sufficient empty space ahead of the robot to allow $R_{0}$ to move forward. We furthermore arrange these projected distances with $d_{o b s, 1}^{\prime}<d_{o b s, 2}^{\prime} \ldots d_{o b s, j}^{\prime}<0 \leq$ $d_{o b s, j+1}^{\prime} \ldots<d_{o b s, N}^{\prime}$ and $d_{r, 1}^{\prime}<d_{r, 2}^{\prime} \ldots d_{r, j}^{\prime}<0 \leq d_{r, j+1}^{\prime} \ldots<d_{r, M}^{\prime}$.

With $d_{r, \text { min }}^{\prime}$ being the $\mathrm{x}$-coordinate of the robot who has the shortest projected distance on the $\mathrm{x}$-axis to robot $R_{0}$ we calculate $d_{o b s, \min }^{\prime}$ according to

$$
d_{o b s, \text { min }}^{\prime}= \begin{cases}d_{o b s, j+1}^{\prime} & \text { if } d_{r, \min }^{\prime}<0 \\ \min \left(\left|d_{o b s, j}^{\prime}\right|,\left|d_{o b s, j+1}^{\prime}\right|\right) & \text { if } d_{r, \text { min }}^{\prime}=0 \\ d_{o b s, j}^{\prime} & \text { if } d_{r, \text { min }}^{\prime}>0\end{cases}
$$

\section{B. Local Decision Making}

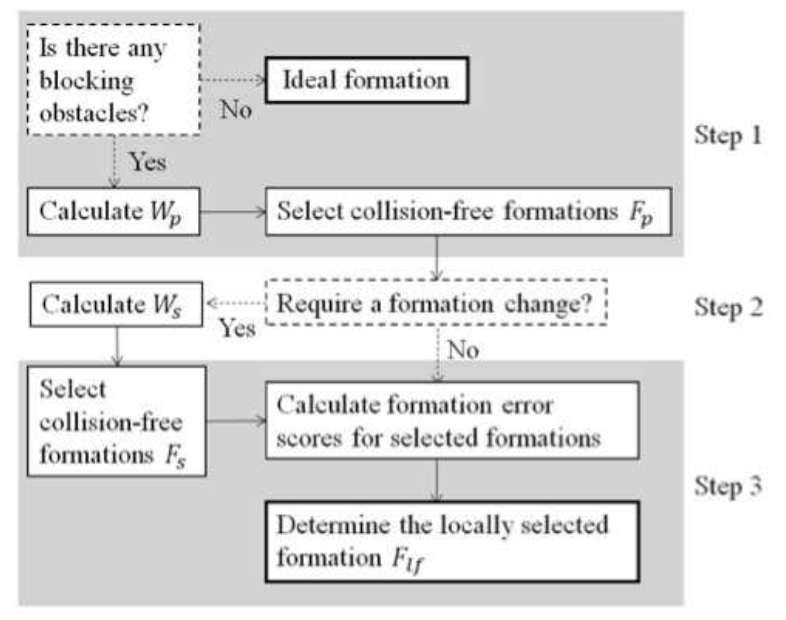

Fig. 3: Overview of Local Decision Making Process

Stage 2 enables each robot to determine if a formation change is required and to select its own locally selected formation out of the collision-free formations. An overview of the process is provided in Fig. 3.

At this stage, the first step is to examine the sensor data from the last stage as indicated in Fig. 3, Step 1. If no obstacle has been detected, the robot will prefer to maintain 
the ideal formation and no further processing is required. In cases where one or more obstacles have been sensed, a formation change may be required to avoid collisions. Alternative formations will be evaluated according to the following criteria:

1) The robot's target destination should be reached without collisions

2) The new formation should introduce the shortest possible travelling distance

3) Unnecessary transitions between alternative formations should be avoided

Alternative formations that only differ from the ideal formation in scale are given higher priority to be evaluated in order to increase the likelihood of preserving the ideal formation shape. Alternative formation are represented by $F_{m, n}$. They are classified by the formation type, or intuitively, the formation shape, $m$ and its associated formation scale $n$. Scaled versions of a formation type is achieved by changing the inter-robot distance $D_{m, n}$. Alternative formations $F_{m, n}$ are evaluated in three steps as described in Fig. 3, the intermediate output formations from the three steps are represented by $F_{p}, F_{s}$ and the final output of this stage, the locally selected formation, is $F_{l f}$.

In Step 1, the parameter $W_{p}$ is calculated for each robot as described in

$$
W_{p}= \begin{cases}\left|d_{o b s, \text { min }}^{\prime}\right|+\left|d_{r, \text { min }}^{\prime}\right| & \text { if } N \neq 0 \\ r_{s}+\left|d_{r, \text { min }}^{\prime}\right| & \text { if } N=0\end{cases}
$$

This parameter measures the projected distance between the closest obstacle and the closest robot to the forward direction vector. As indicated in Step $1, W_{p}$ is used to determine if an alternative formation is collision-free, considering all the surrounding obstacles and robots. The condition for an alternative formation $F_{m, n}$ to be selected in this step as one of $F_{p}$ is

$$
F_{m, n} \in F_{p}, \text { if } D_{m, n} \leq W_{p}-t_{s}
$$

where

$t_{s}:$ Safety distance

Safety distance $t_{s}$ represents the space to be left between the robots and obstacles and is introduced to ensure that the locally selected formation satisfies Criterion 1.

Step 2 checks if all the formations in $F_{p}$ require a change in shape. If no alternative formations which require only a change in scale from the ideal formation can be formed without collisions, a change in formation type is required. In this case, the neighbouring robots change their relative positions, therefore they should not be used as references for the clear path width. Hence the parameter $W_{s}$ is calculated as in

$$
W_{s}=d_{o b s, j+1}^{\prime}-d_{o b s, j}^{\prime}
$$

$W_{s}$ measures the projected distances between the two obstacles that are closest to the forward direction vector, as indicated in Fig. 4.

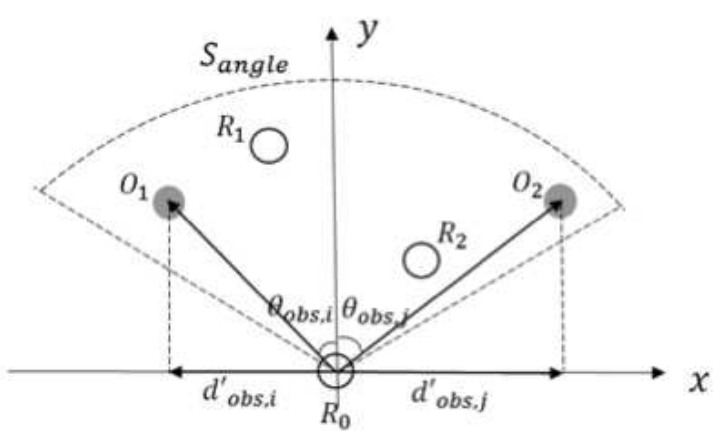

Fig. 4: Calculating the projected distance between the closest obstacles

Then in Step 3, another group of collision free formations $F_{s}$ is selected from

$$
F_{m, n} \in F_{s}, \text { if } D_{m, n} \leq W_{s}-t_{s}
$$

In Step 3, alternative formations are evaluated. If a change in formation type is required, formations in $F_{p}$ are evaluated, if no change in formation type is required, formations in $F_{S}$ are evaluated. In order to satisfy Criterion 2 and Criterion 3, $F_{s}$ or $F_{p}$ are evaluated according to

$$
E_{i}=C_{i} e_{i}
$$

where

$E_{i}$ : Formation error score

$C_{i}$ : Formation state factor

$e_{i}$ : Euclidean distance between current and target positions

The formation with the smallest $E_{i}$ value is selected by the robot to be the locally selected formation $F_{l f}$. Formation state factor $C_{i}$ is introduced to avoid unnecessary transitions, as empirical results show that robots have a tendency to select the more compact formations when only $e_{i}$ is taken into account, even if a more compact formation is required, which in turn results in an unstable and highly fluctuant system. The value for $C_{i}$ is chosen such that $E_{i}$ for the more compact formations is amplified, and they are less likely to be chosen as $F_{l f}$. Also, the value of $C_{i}$ for the ideal formation should be lower than that of the alternative formations in order to amplify its chance of selection.

\section{Distributed Agreement}

Once individual robots have identified locally selected formations, the group as a whole must reach a collective agreement on which formation to select. At this stage, $F_{l f}$ is assigned to the robot as its initial formation state. Given the notation $\xi_{i}[k]$ for formation states of robot $i$ during the collective stage, $\xi_{i}[0]=F_{l f}$. It is proven in [18] that distributed linear iterations offer fast convergence. Consensus is achieved by means of the weighted consensus mechanism in (9). 


$$
\xi_{i}[k+1]=W_{i i} \xi_{i}[k]+\sum_{j \in M} W_{i j} \xi_{j}[k], j=1, \ldots M_{i}
$$

with

$$
\begin{gathered}
W_{i j}=\frac{s_{j} k_{j}}{k_{1}+k_{2}+\ldots+k_{M_{i}}} \\
s_{j}=s_{0} \frac{V_{0}}{V_{j}}
\end{gathered}
$$

where

\section{$k:$ number of iterations}

$\xi_{i}[k]$ : current formation state of robot $i$

$\xi_{i}[k+1]:$ next formation state of robot $i$

$W_{i j}$ : weight on $\xi_{i}$ at robot $i$

$M_{i}$ : number of robots within robot $i$ 's communication radius

$s_{0}:$ step constant

$s_{j}:$ step time of robot $j$

$V_{j}$ : relative robot speed of closest obstacle to robot $j$

$V_{0}:$ standard robot speed

$k_{j}$ : obstacle weight robot $j$ introduced to its surrounding robots

The mechanism is designed such that the closer a robot is to obstacles, the higher its weight will become in the consensus process. Having a weight that is associated with the distance to obstacles exerts a bias on the robots that have more precise information about the surrounding obstacles. The robot that is closest to obstacles is also assumed to have the highest chance of crashing unless the formation is altered and the needs of this robot must therefore be attended to first.

Consensus is reached locally when the state differences of a robot $i$ and its neighbours are smaller than a threshold $T_{s}$. Increasing this threshold will reduce the time it takes to reach consensus. However, if the threshold is too large, the discrepancy in the states will increase. It is therefore important to select a threshold that balances speed and accuracy. We have determined empirically that the state difference threshold should be less than $10 \%$ of the minimum state separation. This recommended threshold is used in Section 3.

Parameter $s_{j}$ is the time interval between two consensus processes, which increases when the speed of robots is reduced with respect to obstacles, and it is therefore less urgent to change formations. Standard relative speed $V_{0}$ is determined according to the recommended speed of specific robots. The benefit of decreasing $s_{j}$ is that consensus can be calculated at a slower pace to reduce energy consumption. It should be noted that there are lower and upper bounds for $s_{j}$. The value should always be larger than the communication round-trip delay to avoid using outdated data. The upper bound can be found empirically by identifying the time that enables the robots to reach consensus when the relative speeds of the robots are close to zero. This upper bound can be further increased if energy preservation is a major
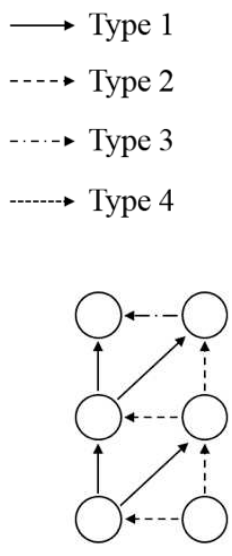

(b) Dual Line

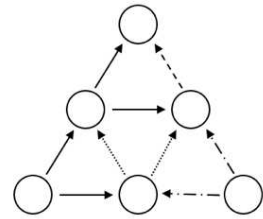

(a) Maximum Coverage

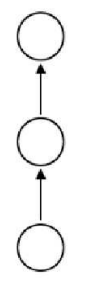

(c) Line
Fig. 5: Alternative formation types

concern, however a higher risk of colliding with obstacles necessitates lower upper bounds. The $k_{j}$ parameter is used to calculate weight $W_{i j}$ and accounts for the distance between obstacles and the robots. This parameter is calculated according to

$$
k_{j}=\exp \left(\frac{\frac{r_{s}}{d_{\min }}-1}{\tau}\right)
$$

where

$d_{\min }:$ distance to closest obstacle, and $0<d_{\min }<r_{s}$

$\tau$ : time constant

\section{EXPERIMENT DESIGN AND RESULTS}

In this section, we validate the proposed formation selection mechanism by means of two experiments: one tests the convergence speed of the proposed consensus algorithm as the relative speed to obstacles increase, while the other evaluates the spatial adaptability to varying path widths. Each experiment was carried out 5 times and the mean of the results were calculated and presented in graphs of this section.

According to [14], [15], [16], several patterns can be used as maximum coverage formations. The optimal pattern depends on the communication and sensing ranges of the robots. Considering the constraints given by our technology and the connectivity requirements in this research, a triangular pattern was selected. The alternative formations used in our experiments are shown in Fig. 5. Different types of arrows between nodes represent different constraint types and each node represent one robot. Correspondingly, Tables II, III and IV display the distance and angle constraints used in each formation. These constraints have been adopted from [6].

In the experiments, two continuous rows of virtual obstacles are driven towards the MRS in order to simulate a narrow tunnel that the robots have to pass through. The formation states held by the robots at every time step are 
TABLE I: Parameters for Implementation Specific Variables

\begin{tabular}{|c|c|}
\hline Parameters & Value \\
\hline$S_{\text {angle }}$ & $180^{\circ}$ \\
\hline$t_{s}$ & $436 \mathrm{~mm}$ \\
\hline$V_{0}$ & $61.2 \mathrm{~mm} / \mathrm{s}$ \\
\hline$T_{s}$ & 0.1 \\
\hline$s_{0}$ & 1 \\
\hline$r_{s}$ & $570 \mathrm{~mm}$ \\
\hline$\tau$ & 0.4 \\
\hline
\end{tabular}

TABLE II: Constraints for Maximum Coverage Formation

\begin{tabular}{|c||c||c||c||c|}
\hline Types & Distance 1 & Angle 1 & Distance 2 & Angle 2 \\
\hline 1 & $r_{s}$ & $30^{\circ}$ & $r_{s}$ & $90^{\circ}$ \\
\hline 2 & $r_{s}$ & $-30^{\circ}$ & - & - \\
\hline 3 & $r_{s}$ & $-30^{\circ}$ & $r_{s}$ & $-90^{\circ}$ \\
\hline 4 & $r_{s}$ & $30^{\circ}$ & $r_{s}$ & $-30^{\circ}$ \\
\hline
\end{tabular}

recorded throughout this process. Fig. 6 shows a scenario in which the MRS selected a Dual Line Formation to allow the robots to pass through the tunnel as the Maximum Coverage Formation, which is used as the ideal formation in our experiments, cannot fit through the narrow path. Since no formation selection algorithms that include obstacle avoidance have been proposed in existing literature, a comparison with other algorithms was not possible.

The MRS consists of five non-holonomic ground moving "eBug" robots [17], while an overhead camera connected to a computer acts as a pseudo-GPS which is used to emulate laser range finders with a $360^{\circ}$ field of view. The computer also generates and moves the virtual obstacles that form the narrow corridor, while ZigBee networks enable the robots to communicate with the computer and neighbouring robots. Implementation specific parameters used by the formation selection algorithm are listed in Table I.

\section{A. Robustness to Increasing Formation Speed}

This experiment evaluated the algorithm's ability to converge to a formation while the relative speed of the robots, with respect to surrounding obstacles, was increased. The

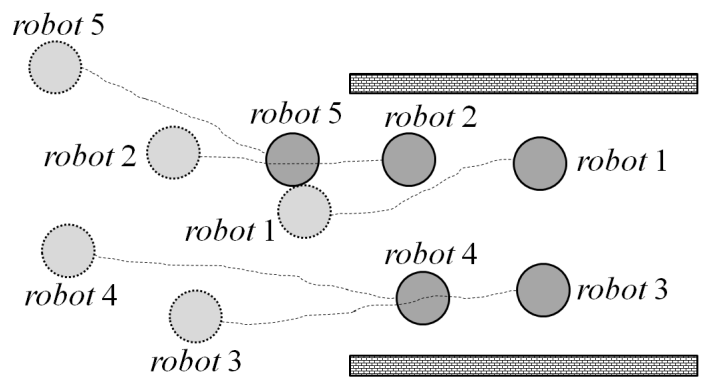

Fig. 6: The path ahead narrows, requiring a change from Maximum Coverage Formation. The MRS determines it is wide enough to accommodate Dual Line Formation, which reduces the total travelling distance comparing to Line Formation.
TABLE III: Constraints for Dual Line Formation

\begin{tabular}{|c||c||c||c||c|}
\hline Types & Distance 1 & Angle 1 & Distance 2 & Angle 2 \\
\hline 1 & $\frac{r_{s}}{\sqrt{2}}$ & $0^{\circ}$ & $r_{s}$ & $45^{\circ}$ \\
\hline 2 & $\frac{r_{s}}{\sqrt{2}}$ & $0^{\circ}$ & $\frac{r_{s}}{\sqrt{2}}$ & $-90^{\circ}$ \\
\hline 3 & $\frac{r_{s}}{\sqrt{2}}$ & $0^{\circ}$ & - & - \\
\hline
\end{tabular}

TABLE IV: Constraints for Line Formation

\begin{tabular}{|c||c||c||c||c|}
\hline Types & Distance 1 & Angle 1 & Distance 2 & Angle 2 \\
\hline 1 & $\frac{r_{s}}{\sqrt{2}}$ & $0^{\circ}$ & - & - \\
\hline
\end{tabular}

collision free path formed by the approaching obstacles was $783.5 \mathrm{~mm}$ wide. Starting from a relative speed of $22.8 \mathrm{~mm} / \mathrm{s}$, samples were taken in increments of $11.4 \mathrm{~mm} / \mathrm{s}$ ending at 228 $\mathrm{mm} / \mathrm{s}$. The results from the experiment are plotted in Fig. 7.

The bottom solid line represents the time taken from when the obstacles are spotted to when the group agrees on a formation, while the dashed line represents the time taken before the robots have moved to their target position in the new formation. One can observe that the algorithm enables the robots to successfully converge to alternative formations, and thereby avoid obstacles, until the break point at 228.0 $\mathrm{mm} / \mathrm{s}$. The relative speed at the breakpoint equals 1.86 times the maximum speed of the robots.

\section{B. Robustness to Narrow Paths}

In this experiment, the stability over various path widths was tested. The separation that allows the formation to pass through the obstacle course was gradually reduced from $661.2 \mathrm{~mm}$ to $233.7 \mathrm{~mm}$, plus a safety distance of $436 \mathrm{~mm}$. Fig. 8 shows the average time taken to reach all intermediate formation types before the final formation was reached.

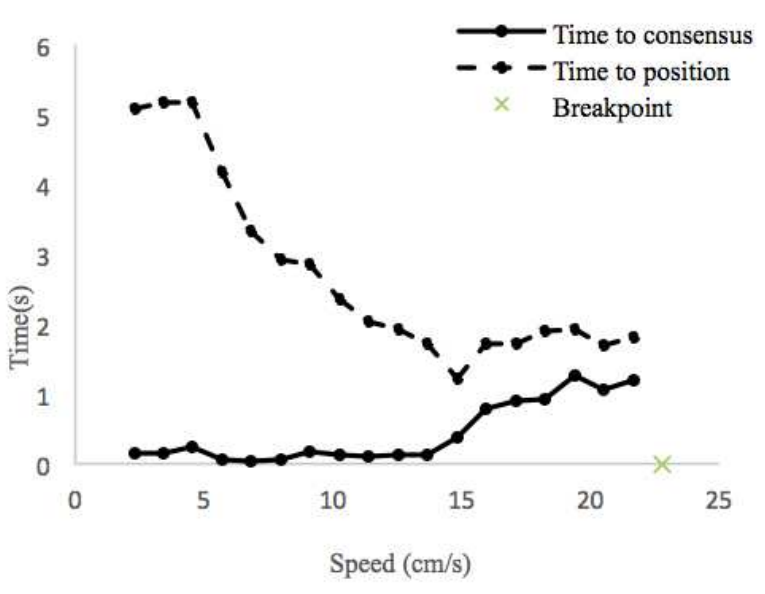

Fig. 7: Time vs. relative speed 


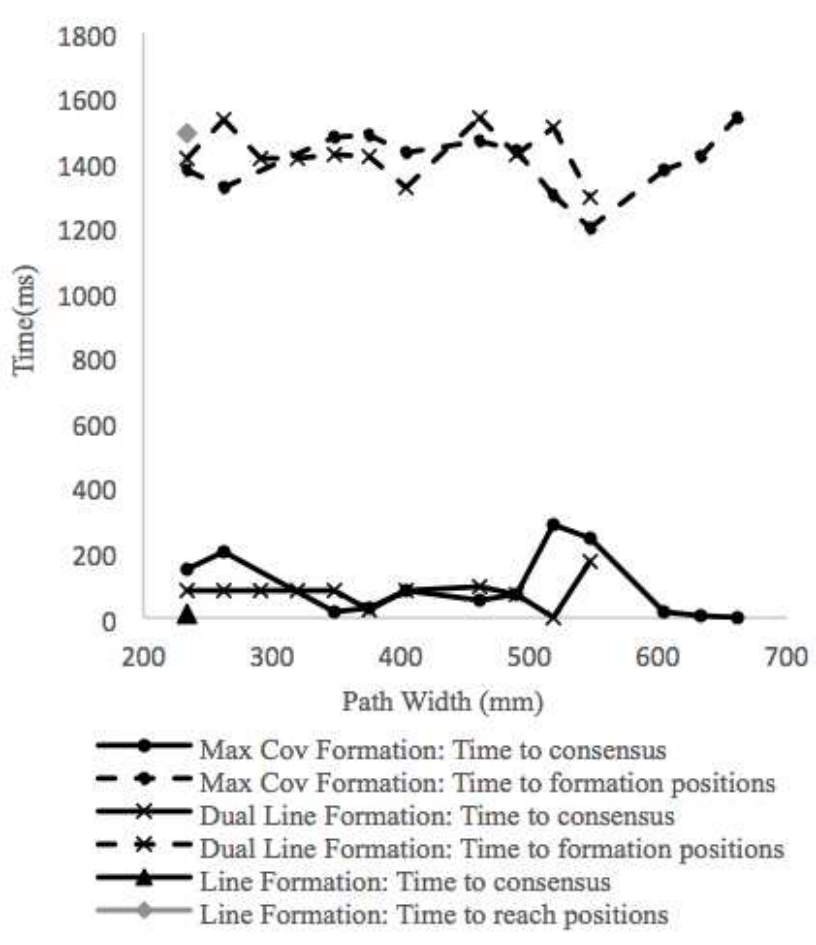

Fig. 8: Time vs. path width

One can observe that the MRS selects its formation according to the path width. The algorithm abandons the maximum coverage formation when the path width is narrower than $400 \mathrm{~mm}$ and thus enables the robots to pass through the obstacle course without experiencing collisions. The line formation is only formed when the path width is $233.7 \mathrm{~mm}$, which demonstrates that the MRS will not converge to a more compact state than is necessary, in order to conserve energy. It should also be noted that the dual line formation is not formed when the path is wider than 547 $\mathrm{mm}$, which justifies the use of formation state factor $C_{i}$.

\section{CONCLUSIONS}

A distributed formation selection algorithm that enables non-holonomic ground moving robots to autonomously select and generate formations was presented in this paper. Experiments conducted on the proposed technology demonstrate that the algorithm enables the robots to navigate through environments with dynamic obstacles without experiencing collisions when the relative speed of the robots with respect to the obstacles is increased to 1.86 times the maximum speed of the robots. The experiments furthermore reveal that the formation selection mechanism is capable of selecting formations without introducing excessive travel distances. Future research can seek modifications in the consensus process to decrease the inaccuracy created by sensor noise in order to increase the system robustness.

\section{ACKNOWLEDGMENT}

S. Yu thanks Daniel Waghorn for his insight and assistance in refining and polishing this paper.

\section{REFERENCES}

[1] Barca, JC. and A. Sekercioglu. Swarm Robotics Reviewed. Robotica, Available on CJO 2012 doi:10.1017/S026357471200032X, pp. 1-32, 2012.

[2] B. Fidan, C. Yu and B. Anderson, Acquiring andmaintaining persistence of autonomous multi-vehicle formations, IET Control Theory Appl. 1(2), pp. 452-460, 2007.

[3] Z. Xue and J. Zeng, Formation Control Numerical Simulations of Geometric Patterns for Unmanned Autonomous Vehicles with Swarm Dynamical Methodologies, Proceedings of the International Conference on Measuring Technology and Mechatronics Automation, Zhangjiajie, China 2009.

[4] M. Mamei, M. Vasari and F. Zambonelli, Experiments of morphogenesis in swarms of simple mobile robots, Appl. Artif. Intell. 218(9-10), pp. 903-919, 2004.

[5] L. Barnes, M. Fields and K. Valavanis, Swarm formation control utilizing elliptical surfaces and limiting functions, IEEE Trans. Syst. Man Cybern. 39(6), pp. 1434-1445, 2009.

[6] Barca, JC., A. Sekercioglu, and A. Ford. Controlling Formations of Robots with Graph Theory. In: S. Lee, Cho, H., Yoon, K. and Lee, J. (eds) Intelligent Autonomous Systems 12. Springer Verlag, pp. 563574, 2013.

[7] G. Podevijn, R. O'Grady, and M. Dorigo. Self-organised Feedback in Human Swarm Interaction, IRIDIA - Technical Report Series, Technical Report No.:TR/IRIDIA/2012-016, pp. 1-6, 2012.

[8] M. Mesbahi and M. Egerstedt (eds.), Graph Theoretic Methods in Multiagent Networks. Applied Mathematics (Princeton University Press, NJ, 2010) pp. 401.

[9] M.A. Haque and M. Egerstedt, Decentralized Formation Selection Mechanisms Inspired by Foraging Bottlenose Dolphins, Mathematical Theory Of Networks and Systems, 2008.

[10] Rubenstein, M.; Wei-Min Shen, Automatic scalable size selection for the shape of a distributed robotic collective, Intelligent Robots and Systems (IROS), 2010 IEEE/RSJ International Conference on , vol., no., pp.508,513, 18-22 Oct. 2010 doi: 10.1109/IROS.2010.5650906.

[11] Seng, L., Barca, J C. and Sekercioglu, A. (2013) Distributed Formation Control in Cluttered Environments. Presented at: 2013 IEEE/ASME International Conference on Advanced Intelligent Mechatronics (AIM).

[12] Meng, Y., Guo, H. and Jin, Y. A morphogenetic approach to flexible and robust shape formation for swarm robotic systems, Robotics and Autonomous Systems. vol 61, pp 25-38, 2013.

[13] Jin, Y., Guo, H. and Meng, Y. A Hierarchical Gene Regulatory Network for Adaptive Multirobot Pattern Formation, IEEE Transactions on Systems, Man and Cybernetics, vol.42, no.3, pp 805-816, 2012.

[14] L Xiao-Yuan LS-B, G Xin-Ping, Automatic generation of minweighted persistent formations, Chinese Physics, pp. B 18(8):31043114, 2009.

[15] Xiaole Bai, Santosh Kumar, Dong Xuan, Ziqiu Yun, Ten H. Lai, Deploying Wireless Sensors to Achieve Both Coverage an Connectivity, MobiHoc, pp. 131-142, 2006.

[16] Ming Ma, Yuanyuan Yang, Adaptive Triangular Deployment Algorithm for unattended Mobile Sensor Networks, IEEE Transactions on Computers, pp. 946-958, 2007.

[17] DAdemo, N., Lui, W. L. D., Li, W. H., Sekercioglu, Y. A., Drummond, T. eBug-An open robotics platform for teaching and research. Proc. ACRA, 2011.

[18] Lin Xiao, Stephen Boyd, Fast Linear Iterations For Distributed Averaging, Conference on Decision and Control, pp.901-906, 2003 